\title{
An III : créer, inventer, réinventer le pouvoir exécutif
}

Christine Le Bozec

\section{Q OpenEdition \\ 1 Journals}

Édition électronique

URL : https://journals.openedition.org/ahrf/824

DOI : 10.4000/ahrf.824

ISSN : 1952-403X

\section{Éditeur :}

Armand Colin, Société des études robespierristes

\section{Édition imprimée}

Date de publication : 1 juin 2003

Pagination : 71-79

ISSN : 0003-4436

\section{Référence électronique}

Christine Le Bozec, "An III : créer, inventer, réinventer le pouvoir exécutif », Annales historiques de la Révolution française [En ligne], 332 | avril-juin 2003, mis en ligne le 22 avril 2008, consulté le 23 avril 2022. URL : http://journals.openedition.org/ahrf/824 ; DOI : https://doi.org/10.4000/ahrf.824

Tous droits réservés 


\title{
AN III : CRÉER, INVENTER, RÉINVENTER LE POUVOIR EXÉCUTIF
}

\author{
CHRISTINE LE BOZEC
}

\begin{abstract}
II s'agit d'une comparaison (fond et forme) des diverses rédactions limitant le pouvoir exécutif dans les trois constitutions (1791, 1793, an III). II a paru nécessaire de faire un détour par le projet présenté en avril 1793 par Boissy d'Anglas puisqu'il est l'un des pères de la Constitution de l'an III. A l'évidence, ce qui se dégage c'est une méfiance, guère dissimulée, envers le pouvoir exécutif.
\end{abstract}

Mots clés : pouvoir exécutif; Constitution de 1791 ; Constitution de 1793 ; projet de constitution de l'an III ; Boissy d'Anglas.

En l'an III, séparer les pouvoirs, c'est une vieille affaire, il n'est nullement question d'y revenir ici, mais de mesurer leur poids respectif. Étudier leurs relations ou leurs interactions éventuelles ainsi que les naissantes responsabilités mutuelles de l'un envers l'autre sera l'objet de notre propos.

Ce travail s'attache à l'étude du pouvoir exécutif dans la Constitution de l'an III, autant à son contenu qu'à sa genèse, en fait à l'itinéraire qui a conduit à sa mise en texte. Cette démarche me semble appeler des comparaisons de type théorique et pratique.

An III : la Commission des Onze réfléchit, rédige et fait adopter par la Convention thermidorienne une nouvelle Constitution. En six ans, c'est la troisième entreprise de ce genre. Depuis l'été 1789, les législateurs ont accouché de trois textes : 1791,1793 et 1795 . À cet endroit, je me pose la question et je pose la question : peut-on inclure la loi du 14 frimaire an II et la considérer telle une constitution provisoire? J'ai fait le choix de seulement l'évoquer et ce afin de rappeler le combat mené pour faire admettre la nécessité de la rédaction d'une nouvelle constitution, en l'an III. 
En premier lieu, il me paraît nécessaire de procéder à quelques comparaisons, points communs et divergences concernant le pouvoir exécutif, entre les trois textes constitutionnels et ce afin de reconstituer litinéraire qui aboutit à la Constitution de 1795.

À chaque étape, c'est-à-dire pour chaque texte, je rappellerai brièvement les conditions de rédaction puis je préciserai l'esprit du texte, c'est-à-dire l'arrière fond théorique qui a présidé à la réflexion et déterminé la mise en mots. Enfin, nous le savons, les conditions d'application sur le terrain, c'est-à-dire la pratique, ont condamné ces textes, souvent trop bel échafaudage, magnifique exercice intellectuel et juridique mais vite balayé par des circonstances qui les rendirent inexorablement obsolètes.

Je ne me contenterai pas de ces trois textes au caractère officiel parce que approuvés, votés et promulgués. Je désire me référer, en conclusion, à l'un des trois cents projets répondant à l'appel de Barère du 19 octobre 1792, proposant à «tous les amis de la liberté et de l'égalité » de soumettre à la Convention leurs idées et leurs lumières. On peut le considérer comme un des projets les plus aboutis. Celui-ci fut annoncé dans le Journal des Débats et décrets du 13 avril 1793, et présenté le 17 avril 1793 à la Convention par son auteur, le futur rapporteur de la Commission des Onze (1), le conventionnel Boissy d'Anglas (2). Quel hasard !

Grâce à ce projet, il s'agit pour moi de montrer le texte de l'an III et dans sa réflexion et dans sa rédaction comme un aboutissement, situé dans le droit fil d'une pensée juridique et libérale bien antérieure à l'an III mais plus élaborée que celle qui préside à la rédaction de la Constitution de 1791. En 1793, cette pensée était dans l'air mais elle ne pouvait s'exprimer, en revanche après le 9 thermidor, elle le put.

De fait, les trois textes présentent des points communs nombreux :

- une absence de parlementarisme au sens moderne du terme ;

- une domination ou une nette prédominance du pouvoir législatif;

- un pouvoir exécutif contrôlé et après 1792 pluricéphale ;

- un pouvoir exécutif ne possédant aucune initiative des lois, il les fait exécuter, mais ne peut ni les modifier, ni les proposer ;

- un lieu de résidence défini, pour le pouvoir législatif, très proche du siège du pouvoir législatif;

-une liste civile, un traitement ou des émoluments plus que convenables ;

(1) Cf. Christine LE BOZEC, «Le républicanisme du possible », Annales historiques de la Révolution française, $1995, \mathrm{n}^{\circ} 1$.

(2) Cf. Christine LE BOZEC, « Sur quelque projet constitutionnel », dans L'an I et l'apprentissage de la démocratie, Saint-Denis, 1993, Éditions PSD, Saint-Denis, 1995. 
- si la force armée lui est confiée, c'est avec méfiance et de nombreuses précautions sont prises ainsi que des garde-fous mis en place ;

- la Trésorerie lui échappe.

Il est intéressant d'insister sur l'énoncé du texte de 1791, pour constater à quel point l'on se garantit contre l'arbitraire royal. En effet on retrouve des termes contenus dans les cahiers de doléances encore tout proches. Ils révèlent les inquiétudes, les refus, les indignations que la fiscalité et son fonctionnement ont soulevés au XVIII ${ }^{\mathrm{e}}$ siècle. L'Exécutif ne surveille que la perception, la création, le montant et la répartition des impôts passent sous la responsabilité du Législatif. L'insistance dans l'énoncé montre à quel point les rédacteurs demeurent sous la crainte des abus de l'Ancien Régime.

Les différences, quant à elles sont moins nombreuses :

- l'on passe d'un cadre monarchique à celui républicain ;

- on peut noter la faiblesse ou l'importance du nombre des articles concernant le pouvoir exécutif, mais aussi constater la sécheresse ou la prolixité de ceux-ci ;

- enfin se précise un retour vers ou un renforcement de la centralisation, de fait un très net contrôle de l'État central à partir de 1793.

Caractères généraux, différences, je développerai, pour chacune d'entre elles, quelques traits caractéristiques. La Constitution de 1791 fut le fait d'hommes neufs, politiquement s'entend, même si parmi eux nombreux étaient les juristes rompus à la réflexion et à la rédaction. Ces hommes étaient fils des Lumières et de l'Ancien Régime. Ceci est très prégnant dans leurs considérations, leurs énoncés et leurs préventions. Rappelons qu'un premier comité de trente membres fut désigné le 6 juillet 1789 pour rédiger une constitution. Au lendemain du 14 Juillet, un nouveau comité fut constitué. Le 27 juillet, la Constituante décida de la rédaction d'une Déclaration des droits de l'homme et du citoyen. Le 4 août, les députés annoncèrent que cette déclaration précéderait le texte constitutionnel.

Dans le texte, la place attribuée à l'Exécutif, la disposition et le nombre des articles, soixante-deux, montrent à quel point on désire se détacher de l'Ancien Régime mais l'on baigne encore dans son vocabulaire et ses craintes. On passe de la théorie rêvée dans les libelles, les journaux et les cahiers à une pratique sur le terrain. La situation est nouvelle. Ainsi dans le texte, la place du pouvoir exécutif ne se détache pas avec une réelle netteté, il y règne une certaine confusion.

$\mathrm{Si}$ « de l'exercice du pouvoir exécutif » constitue un bloc compact, que l'article 6 de la section $1^{\mathrm{re}}$ du chapitre IV caractérise : «Le pouvoir exécutif ne peut faire aucune loi, même provisoire, mais seulement des proclamations conformes aux lois, pour en ordonner ou en rappeler l'exécution», auparavant, disséminé dans le texte, on trouve : «De la sanction royale », 
«Des relations du Corps législatif avec le roi» après avoir auparavant longuement défini : «De la royauté et du roi », «De la régence», « De la famille du roi», «Des ministres». Donc un ensemble de soixante-deux articles, parfois répétitifs et qui se croisent et s'entrecroisent. Cette réflexion qui peut paraître donner beaucoup d'importance au côté formel me semble primordiale parce qu'ils révèlent bien des hésitations, des balbutiements, des bégaiements, un laborieux apprentissage, la forme révélant le fond.

Soulignons la réelle particularité, concernant ce pouvoir, que constitue l'instauration du veto royal, moyen élégant de contourner l'autorité royale mais surtout de paraître ne pas vouloir l'éliminer complètement, veto dont nous savons combien il fut paralysant, une véritable gangue qui bloqua le fonctionnement des institutions. L'article 2 de la section III du chapitre III stipule : «Dans le cas où le roi refuse son consentement, ce refus n'est que suspensif. Lorsque les deux législatures qui suivront celle qui aura présenté le décret, auront successivement représenté le même décret dans les mêmes termes, le roi sera censé avoir donné la sanction». Le 10 Août trancha ce nœud gordien et conduisit à rédiger un nouveau texte, la Constitution de 1793.

En 1793, la Convention, terme inspiré par l'organisation constitutionnelle américaine, est chargée de rédiger une nouvelle constitution, c'est le but premier assigné à cette nouvelle Assemblée.

Des rivalités politiques Girondins/Montagnards, un arrière-fond de guerre extérieure, intérieure puis enfin une forme de précipitation pour le boucler le 24 juin 1793 ont présidé à la rédaction du texte. Il ne faut pas omettre les trois cents contributions proposées par les citoyens. À l'occasion, profitons-en pour mentionner les quelques voix rares et timides qui ont fait une allusion discrète au suffrage féminin.

Si le texte constitutionnel est bref, la place attribuée au pouvoir exécutif est plus que réduite : quinze articles très courts, très secs, noyés dans le texte. Ils déterminent un Exécutif faible, on a envie de dire affaibli, contrôlé, muselé, composé de vingt-quatre membres. L'assemblée électorale de chaque département nomme un candidat, c'est alors le Corps législatif qui choisit les vingt-quatre membres, le Conseil exécutif, renouvelé par moitié à chaque législature, c'est-à-dire tous les ans.

Dans ses attributions, il apparaît plus de restrictions que de possibles initiatives. Parler de sa négation serait peut-être exagéré, il faudrait plutôt évoquer les craintes infinies qu'il semble inspirer aux rédacteurs. En effet, le Conseil exécutif n'est habilité qu'à l'exécution des lois et des décrets du Corps législatif, il est responsable de l'inexécution des lois et ne peut être entendu que lorsqu'il a des comptes à rendre. Le Corps législatif l'appelle lorsqu'il le juge convenable et met en accusation ses membres en cas de prévarication. La sécheresse de l'article 65 caractérise parfaitement les 
articles concernant l'Exécutif : «Le Conseil est chargé de la direction et la surveillance de l'administration générale; il ne peut agir qu'en exécution des lois et des décrets du Corps législatif».

Il doit nommer les agents en chef de l'administration générale de l'administration, dont le nombre est déterminé par le Corps législatif, en dehors de son sein. C'est lui qui révoque et remplace les agents à sa nomination, qu'il doit dénoncer en cas de besoin. S'il négocie les traités, il ne les signe pas. Un Exécutif surveillé, réduit, encadré et privé de toute initiative par un Corps législatif puissant et s'il semble issu du peuple, il y a tout de même un choix ultime effectué par le Législatif que précise l'article 63 : «L'Assemblée électorale de chaque département nomme un candidat. Le Corps législatif choisit, sur la liste générale, les membres du Conseil ».

Nous savons que cette Constitution ne fut pas appliquée et que la pratique de l'an II en infirma l'esprit puisque les principes du gouvernement révolutionnaire définis le 14 frimaire an II (4 décembre 1793), cette "constitution provisoire », ce «gouvernement exceptionnel imposé par les circonstances» mirent en place deux conseils, le Comité de Salut public et le Comité de sûreté générale, c'est-à-dire la dictature d'un pouvoir exécutif composé d'hommes issus directement du corps législatif, donc de l'élection populaire. Les circonstances avaient engagé l'exercice du pouvoir et des pouvoirs sur des voies et des rapports nouveaux et originaux. C'est de cette pratique dont voulurent se défaire les Thermidoriens de l'an III.

En 1795, la question qui se pose est : que faire du Gouvernement révolutionnaire ? On vida rapidement les Comités de leur contenu, on les dessaisit de leurs attributions et après avoir tergiversé et pensé adjoindre quelques articles organiques à la Constitution de 1793, la peur sociale provoquée par Germinal puis par Prairial détermina les Conventionnels à accepter la rédaction d'un nouveau texte constitutionnel. La Commission des Onze fut nommée à cet effet. Sa composition fut soigneusement et stratégiquement dosée (3).

Entre les quinze courts articles de 1793 et les soixante-deux, parfois alambiqués, de 1791, le rôle du pouvoir exécutif dans celle de 1795 est développé dans quarante et un articles dont la longueur est variable, mais le contenu de ces articles est plutôt nourri.

Si le pouvoir exécutif constitué par cinq directeurs, âgés d'au moins quarante ans est surveillé, il est moins encadré, moins étouffé, j'aurai envie de dire moins nié que dans la Constitution précédente. 
Art. 132, titre VI : «Le pouvoir exécutif est délégué à un Directoire de cinq membres, nommé par le Corps législatif, faisant alors les fonctions d'Assemblée électorale au nom de la Nation ».

Le texte qui instaure le renouvellement d'un membre chaque année, pose des limites familiales précises afin d'éviter toute tentation népotique : «L'ascendant et le descendant en ligne directe, les frères, l'oncle et le neveu, les cousins au premier degré, et les alliés à ces divers degrés, ne peuvent être en même temps membres du Directoire, ni s'y succéder, qu'après un intervalle de cinq ans » (art. 139, titre VI).

Ce texte empêche la réélection d'un directeur pendant cinq ans, renouvelle la présidence tous les trois mois, lui autorise la nomination de six à huit ministres, âgés d'au moins trente ans, celle de quatre messagers d'État qu'il peut destituer, et la désignation, hors de son sein, d'un secrétaire, lui interdit de délibérer à moins de trois directeurs présents, le rend responsable de l'exécution des lois, l'autorise à proposer des projets tout en lui refusant une quelconque initiative des lois, l'oblige à résider dans la même commune que le Corps législatif.

S'il dispose de la force armée, il ne la commande pas, il nomme les généraux (avec toujours les mêmes précautions familiales), ne décide pas de la guerre mais la conduit en la confiant aux militaires, négocie les traités qui n'ont de valeur qu'une fois ratifiés par le Législatif. En revanche certaines conditions secrètes relèvent du Directoire.

Il nomme tous les fonctionnaires sauf dans les colonies des îles de France et de La Réunion, enfin le Corps législatif peut donner son autorisation à la nomination d'agents particuliers par le Directoire.

De fait, chaque détenteur du pouvoir exécutif, chaque Directeur est honoré, gardé, largement rétribué, somptueusement logé (art. 165, 166, 167, $168,169,172,173)$, mais ses faits et gestes restent très surveillés, de plus il doit justifier de sa résidence deux ans après la fin de son mandat. L'homme semble plus surveillé que la fonction. L'article 164, titre VI resserre cette surveillance : «Aucun membre du Directoire ne peut s'absenter plus de cinq jours, ni s'éloigner au-delà de quatre myriamètres (huit lieues moyennes) du lieu de la résidence du Directoire, sans l'autorisation du Corps législatif».

Ce texte compliqué, touffu, précautionneux, méfiant, très juridique et intellectuel, très difficile à faire fonctionner dans la pratique, les coups d'État le montrent à l'envi, était une «machine de guerre » (4) qui ne cherchait qu'à se prémunir contre toute tentative de pouvoir personnel, désirait écarter le danger royaliste et encore plus redoutait un retour à l'« anarchie terroriste » ou celui de la pression populaire. Dans les faits : un Exécutif guère issu du peuple, surveillé parce que craint.

(4) Selon la formule célèbre de Marcel Reinhardt. 
Mais ce texte n'est pas brusquement sorti d'un chapeau et la peur sociale qui a favorisé sa rédaction n'est pas que la conséquence de Prairial même si cet événement fut déterminant. J'ai dit précédemment que son inspirateur et son rapporteur à la Commission des Onze fut Boissy d'Anglas. Or celui-ci avait présenté en avril 1793 un projet tout aussi compliqué que l'on ne peut ni négliger ni escamoter car sa construction, son contenu et son vocabulaire préfigurent, annoncent la Constitution de l'an III.

Il est vrai que les circonstances d'avril 1793 interdisaient certaines propositions et que l'auteur, un brillant juriste, a su habilement en masquer l'esprit réel pour présenter un texte qui, nous sommes en 1793, renvoie une première image démocratique mais qu'il faut lire entre les lignes et entre les mots avec attention, méfiance et précaution. Un juriste habile qui savait le sens des mots, mais aussi leur sens caché, qui savait faire dans un implicite que l'on retrouve largement explicité en 1795. Il n'y a pas de rupture dans la pensée mais en 1793, elle sait faire souterraine sa continuité. Dans le projet de Boissy d'Anglas, il y a la Constitution de l'an III en gestation. Il lui (leur) fallait patienter.

Le projet de Boissy d'Anglas était très compliqué, aussi compliqué que celui de l'an III. De nombreuses barrières, d'importants garde-fous visaient à éviter la tyrannie, le pouvoir personnel ou celui d'une assemblée. Il écarterait élégamment et rapidement par sa complication en la lassant et en la décourageant la participation populaire. Un projet libéral habilement masqué, dicté par les circonstances mais il est fondamental de constater lorsqu'on l'étudie que derrière un vocabulaire démocratique, il ne lâche rien de la pensée libérale en la maquillant. On est dans le non-dit. On y décèle aussi la même crainte de l'Exécutif, la même volonté d'encadrement de celui-ci.

Le contenu de ce projet étant très peu connu, il nous paraît utile d'en présenter de larges extraits concernant le pouvoir exécutif. Ces articles constituent le Titre VI, section première : «Du Conseil exécutif», section II «Des relations du Conseil exécutif avec l'Assemblée législative» et la section III : «De l'élection des membres du Conseil exécutif national». Lorsque cela n'est pas précisé dans le texte qui suit, il s'agit de la section I.

Art. $1^{\text {er }}$ : «Il y aura un conseil exécutif national, seul et unique pour toute la République française ».

Art. 2 : «Le Conseil exécutif sera composé de quinze membres et d'un secrétaire général élu à temps par le peuple, conformément au mode d'élection qui sera ci-après déterminé ».

Ainsi ce Conseil exécutif national était élu pour quatre ans par un collège d'électeurs. Ces grands électeurs à l'américaine, eux-mêmes élus par un corps électoral disséminé dans trois villes distantes de cinquante lieues au moins du Corps législatif pour éviter toute pression, élisaient ces 
membres renouvelables par moitié. Ceux-ci constituaient une autorité une et indivisible, un secrétaire général leur était adjoint. En cas d'absence de majorité, on envisageait jusqu'à trois tours choisissant sur une liste complémentaire pour atteindre les quinze nécessaires (définis dans les vingt-deux articles de la section III du Titre VI : « De l'élection des membres du Conseil exécutif national »).

Art. 3 : «D sera présidé alternativement par un de ses membres. Tous les quinze jours, il sera procédé à cet effet, dans ce conseil, à une élection nouvelle, et nul ne pourra être élu président plus d'une fois dans le même trimestre ».

Art. $4:$ : [...] Tout les deux ans, il en sera réélu la moitié. La première fois ceux qui devront être réélus seront désignés par la voie du sort ».

Le corps électoral qui élit ce Conseil ne possède «aucun caractère de représentation publique » (art. 20, section III), en cas de faute, fraude électorale, c'est le Corps législatif qui tranche. Le projet préconise une procédure lourde, très compliquée, longue, totalement coupée de tout électorat populaire et pas plus issue du peuple que les autres textes.

Art. 19 : [...] Le Conseil exécutif se choisira trente adjoints dont deux seront attachés à chacun de ses membres [...] ils assisteront à ses séances, mais sans voix délibératives, et ils seront chargés de l'exécution de ses divers actes ».

Ces adjoints sont nommés pour deux ans (art. 20), à la majorité absolue des voix et il est prévu qu'à partir de 1800 , «nul ne pourra être élu membre du Conseil sans avoir été adjoint pendant deux ans »(art. 21). Des adjoints donc mais pas de ministre dans ce projet.

Art. 16 : «Le titre de ministre est aboli, ainsi que les divisions ministérielles. L'autorité du Conseil est une et indivisible; il n'y aura parmi les membres qui le composent aucune distinction de département [...]».

Art. 24 : « Le traitement des membres du Conseil sera de 20000 livres; celui du secrétaire général de 15000 livres, et celui des adjoints de 10000 livres ».

La Trésorerie échappe à l'Exécutif (art. 17-18) mais «les ordres de paiement seront arrêtés au nom du Conseil et donnés en son nom» (section II, art. 1, 2, 3).

Les articles 5, 6, 7 envisagent que ce Conseil exécutif promulgue, exécute les lois, mais ne peut ni les modifier ni les interpréter, il est autorisé à des proclamations pour rappeler à leur bonne exécution. Il peut suspendre mais pas destituer les administrateurs, le Législatif s'en occupe, mais il peut rappeler ou destituer les administrateurs civils et militaires qui sont nommés par lui » (art. 10).

Art. 8 : «Il ne pourra mettre en jugement aucun fonctionnaire public, mais il sera tenu de dénoncer au Corps législatif les prévarications qui pourraient être commises ». 
Art. 9 : «Tous les agents de l'administration et du gouvernement dans toutes ses parties, sont essentiellement subordonnés au Conseil exécutif; mais l'administration de la justice est seulement soumise à sa surveillance ».

Les armée de Terre et Mer dépendent de lui (art. 11), mais l'article 12 précise : «Mais l'Assemblée législative peut le charger spécialement de prendre en considération telle ou telle mesure de sûreté générale, et il est tenu de rendre compte de ses délibérations à ce sujet ».

L'article 14 lui autorise la nomination des ambassadeurs mais le Conseil se contente de proposer les traités et les alliances.

Enfin Le Conseil peut être invité à rendre compte devant le Législatif, mais en aucun cas il ne donne son avis sur les décisions prises par le Corps législatif à moins d'y être officiellement invité, celui-ci ne peut correspondre qu'avec le président du Conseil (section II, art. 4-5).

Ce projet, à quelques mots et nuances près, annonce ce que propose « L'homme-orchestre de l'an III », « L'homme de la Constitution de l'an III », le «Thermidorien triomphant» (5), de fait porte-parole de la bourgeoisie libérale, enfin au pouvoir. Et c'est ce qu'entérinent les Thermidoriens : «Après thermidor, l'implicite peut devenir explicite » (6). Le but poursuivi depuis 1789 - mise en place d'une classe politique, d'un groupe de spécialistes défendant la suprématie d'une société bourgeoise qui peut se passer de l'intervention et des pratiques utilisées par les masses populaires pour sauver les acquis révolutionnaires de 1789-1791 - semble désormais atteignable.

Mais des précautions à prendre demeurent. Si elles semblent discrètement «prises en compte» par ce projet de 1793, en revanche lorsque Boissy d'Anglas siège à la Commission des Onze, il peut alors les négliger et faire passer en force ce qu'il n'osait deux années auparavant. L'on retrouve dans le projet de 1793 et dans le texte constitutionnel les dispositions identiques qui permettent d'éliminer une grande partie de l'électorat et qui peuvent décourager et lasser celui potentiel.

De la même manière ces textes, réalisations et projets, se garantissaient tous de la crainte de ne pouvoir contrôler un pouvoir exécutif, qu'il soit monarchique ou pluricéphale. En suivant un parcours, apparemment sinueux et chaotique, ils présentent tous la même continuité dans ce dessein.

Christine LE BOZEC

Université de Rouen

Les hauts de Saint-André

82, rue du Maréchal Gallieni

76130 Mont-Saint-Aignan

(5) Christine LE BOZEC, Boissy d'Anglas, un grand notable libéral, Privas, FOL, 1995 (publication de la thèse, dirigée par C. Mazauric, soutenue à l'Université de Rouen en 1993).

(6) Christine LE BOZEC, « Sur quelque projet constitutionnel », op. cil. 